Article

www.mdpi.com/journal/ijms

\title{
Schultz Index of Armchair Polyhex Nanotubes
}

\section{Mehdi Eliasi * and Nafiseh Salehi}

Islamic Azad University Najafabad Branch, Isfahan, Iran; E-Mail: nsalehi@iaun.ac.ir

^ Author to whom correspondence should be addressed. E-mail: eliasi@ math.iut.ac.ir

Received: 3 July 2008; in revised form: 23 August 2008 / Accepted: 14 October 2008 / Published: 29 October 2008

\begin{abstract}
The study of topological indices - graph invariants that can be used for describing and predicting physicochemical or pharmacological properties of organic compounds - is currently one of the most active research fields in chemical graph theory. In this paper we study the Schultz index and find a relation with the Wiener index of the armchair polyhex nanotubes $T U V C_{6}[2 p, q]$. An exact expression for Schultz index of this molecule is also found.
\end{abstract}

Keywords: Topological index; Wiener index; Schultz index; Armchair nanotube; Molecular graph; Distance; Carbon Nanotube.

\section{Introduction}

Topological indices are a convenient method of translating chemical constitution into numerical values that can be used for correlations with physical, chemical or biological properties. This method has been introduced by Harold Wiener as a descriptor for explaining the boiling points of paraffins [1-3]. If $d(u, v)$ is the distance of the vertices $u$ and $v$ of the undirected connected graph $G$ (i.e., the number of edges in the shortest path that connects $u$ and $v$ ) and $V(G)$ is the vertex set of $G$, then the Wiener index of $G$ is the half sum of distances over all its vertex pairs $(u, v)$ :

$$
W(G)=\frac{1}{2} \sum_{u \in V(G)} \sum_{v \in V(G)} d(u, v) .
$$

A unified approach to the Wiener topological index and its various recent modifications is presented. Among these modifications particular attention is paid to the Hyper-Wiener, Harary, Szeged, Cluj and 
Schultz indices as well as their numerous variants and generalizations [4-10]. The Schultz index of the graph $G$ was introduced by Schultz [14] in 1989 and is defined as follows:

$$
S(G)=\frac{1}{2} \sum_{u \in V(G)} \sum_{v \in V(G)}(\operatorname{deg}(u)+\operatorname{deg}(v)) d(u, v),
$$

where $\operatorname{deg}(u)$ is the degree of the vertex $u$.

The main chemical applications and mathematical properties of this index were established in a series of studies [12-15]. Also a comparative study of molecular descriptors showed that the Schultz index and Wiener index are mutually related [16-18].

Carbon nanotubes, the one-dimensional carbon allotropes, are intensively studied with respect to their promise to exhibit unique physical properties: mechanical, optical electronic etc. [19-21]. In [19], Diudea et al. obtained the Wiener index of $T U V C_{6}[2 p, q]$, the armchair polyhex nanotube (see Figure 1). Here we find a relation between the Schultz index and Wiener index of this molecule. By using this relation we find an exact expression for the Schultz index of the same. The Appendix includes a Maple program [22] to produce the graph of $T U V C_{6}[2 p, q]$, and to compute the Schultz index of the graph.

\section{Schultz index of armchair polyhex nanotubes}

Throughout this paper $G:=T U V C_{6}[2 p, q]$ denotes an arbitrary armchair polyhex nanotube in terms of its circumference $2 p$ and their length $q$, see Figure 2. At first we consider an armchair lattice and choose a coordinate label for it, as illustrated in Figure 2. The distance of a vertex $u$ of $G$ is defined as

$$
d(u)=\sum_{x \in V(G)} d(u, x)
$$

the summation of distances between $v$ and all vertices of $G$. By considering this notation the following lemma gives us a relation between the Schultz and Wiener index of $G$.

Figure 1. A $T U V C_{6}[2 p, q]$ Lattice with $p=5$ and $q=7$.

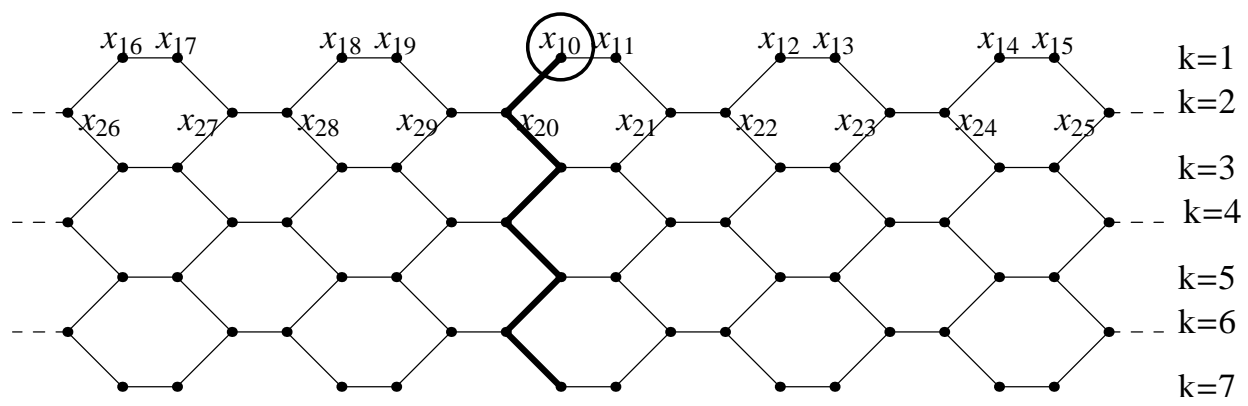

Lemma 1. For the graph $G=T U V C_{6}[2 p, q]$ we have

$$
S(G)=6 W(G)-2 \sum_{u \in \text { level } 1} d(u) .
$$


Figure 2. An armchair polyhex nanotube [19].

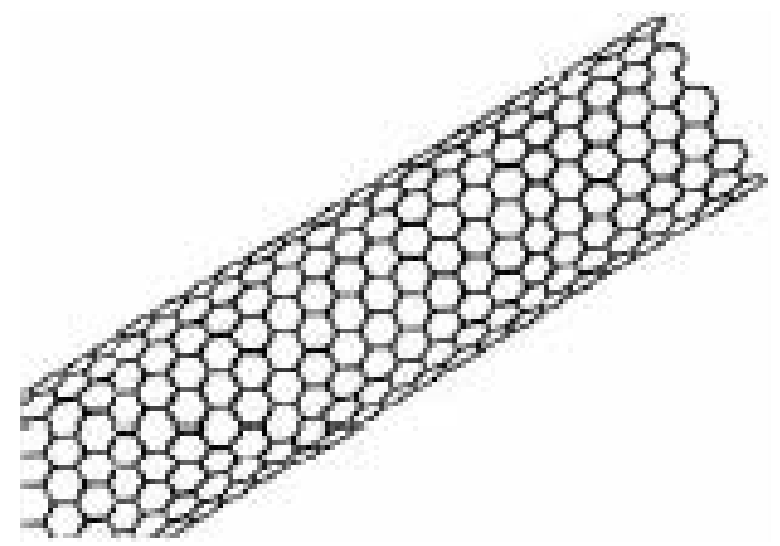

Figure 3. Distances from $x_{01}$ to all vertices of $T U V C_{6}[2 p, q]$ with $p=5$ and $q=7$.

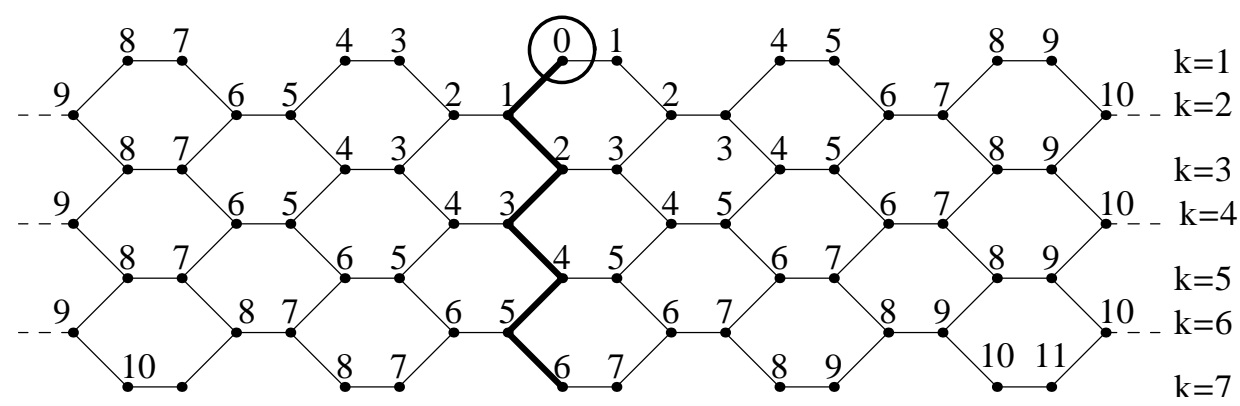

Proof: For each $k$ such that $1 \leq k \leq q$ put $A_{k}:=\{u \in V(G) \mid u \in$ level $k\}$ ( see Figure 2). Then

$$
\begin{aligned}
S(G) & =\frac{1}{2} \sum_{u \in V(G)} \sum_{v \in V(G)}(\operatorname{deg}(u)+\operatorname{deg}(v)) d(u, v) \\
& =\frac{1}{2} \sum_{u \in V(G)} \sum_{v \in V(G)} \operatorname{deg}(u) d(u, v)+\frac{1}{2} \sum_{u \in V(G)} \sum_{v \in V(G)} \operatorname{deg}(v) d(u, v) \\
& =\frac{1}{2} \sum_{u \in V(G)} \sum_{v \in V(G)} \operatorname{deg}(u) d(u, v)+\frac{1}{2} \sum_{v \in V(G)} \sum_{u \in V(G)} \operatorname{deg}(v) d(u, v) \\
& =\frac{1}{2} \sum_{u \in V(G)} \operatorname{deg}(u) \sum_{v \in V(G)} d(u, v)+\frac{1}{2} \sum_{v \in V(G)} \operatorname{deg}(v) \sum_{u \in V(G)} d(u, v) \\
& =\frac{1}{2} \sum_{u \in V(G)} \operatorname{deg}(u) d(u)+\frac{1}{2} \sum_{v \in V(G)} \operatorname{deg}(v) d(v) \\
& =\sum_{u \in V(G)} \operatorname{deg}(u) d(u)
\end{aligned}
$$


But

$$
\operatorname{deg}(u)= \begin{cases}2 & \text { if } u \in A_{1} \cup A_{q} \\ 3 & \text { if otherwise. }\end{cases}
$$

Also in the graph $G$ it is clear that $\sum_{u \in A_{1}} d(u)=\sum_{u \in A_{q}} d(u)$. Therefore

$$
\begin{aligned}
S(G) & =\sum_{u \in V(G)} \operatorname{deg}(u) d(u)=\sum_{u \in A_{1} \cup A_{q}} \operatorname{deg}(u) d(u)+\sum_{u \in V(G) \backslash\left(A_{1} \cup A_{q}\right)} \operatorname{deg}(u) d(u) \\
& =\sum_{u \in A_{1} \cup A_{q}} 2 d(u)+\sum_{u \in V(G) \backslash\left(A_{1} \cup A_{q}\right)} 3 d(u) \\
& =3 \sum_{u \in V(G)} d(u)-2 \sum_{u \in A_{1}} d(u) \\
& =6 W(G)-2 \sum_{u \in A_{1}} d(u) .
\end{aligned}
$$

This completes the proof.

To compute the $d(u)$ in the graph $G$, when $u$ is a vertex in level 1, we first prove the following lemma.

Lemma 2. The sum of distances of one vertex of level 1 to all vertices of level $k$ is given by

$$
\begin{aligned}
w_{k}:=\sum_{x \in \text { level } k} d\left(x_{10}, x\right) & =\sum_{x \in \text { level } k} d\left(x_{11}, x\right) \\
& \vdots \\
& = \begin{cases}2 p^{2}+k^{2}-2 k-2 p+1+H(p, k) & \text { if } 1 \leq k<p \\
p(p+2 k-2) & \text { if } k \geq p,\end{cases}
\end{aligned}
$$

where

$$
H(p, k)= \begin{cases}2 p-1 & \text { if } k+p \text { is even } \\ 2 p & \text { if } k+p \text { is odd }\end{cases}
$$

Proof: We calculate the value of $w_{k}$. We consider that the tube can be built up from two halves collapsing at the polygon line joining $x_{10}$ to $x_{q, 0}$ (see Figure 2). The right part is the graph $G_{1}$ which consists of vertical polygon lines $0,1, \ldots, p$ and $x_{10}$ is one of the vertices in the first row of the graph $G_{1}$. The left part is the graph $G_{2}$ which consists of vertical polygon lines $(p+1),(p+2), \ldots, 2 p-1$. We change the indices of the vertices of $G_{2}$ in the following way:

$$
V\left(G_{2}\right)=\left\{\hat{x}_{j i} \mid \hat{x}_{j, i}=x_{j, 2 p-i} \in V(G)\right\}
$$

(See Figure 3)

We must consider two cases:

Case 1: If $k \geq p$. In the graphs $G_{1}$ and for $0 \leq i<k$ we have

$$
d\left(x_{10}, x_{k, i}\right)=k+i-1 .
$$


Also in the graphs $G_{2}$ and for $1 \leq i<k$ we have

$$
d\left(x_{10}, \hat{x}_{k, i}\right)=k+i-1
$$

So

$$
\sum_{x \in \text { level } k} d\left(x_{10}, x\right)=2 \sum_{i=1}^{p-1}(k+i-1)+(0+k-1)+(p+k-1)=p(p+2 k-2)
$$

Case 2: If $k<p$. First suppose that $1 \leq i<k$. In the graphs $G_{1}$ and $G_{2}$ we have

$$
d\left(x_{10}, x_{k, i}\right)=k+i-1=d\left(x_{10}, \hat{x}_{k, i}\right)=k+i-1 .
$$

Now suppose that $k \leq i \leq p$. Then in the graph $G_{1}$ we can see that if $k$ is odd, then

$$
d\left(x_{10}, x_{k, i}\right)= \begin{cases}2 i & \text { if } i \text { is even } \\ 2 i-1 & \text { if } i \text { is odd }\end{cases}
$$

and if $k$ is even, then

$$
d\left(x_{10}, x_{k, i}\right)= \begin{cases}2 i-1 & \text { if } i \text { is even } \\ 2 i & \text { if } i \text { is odd. }\end{cases}
$$

Also in $G_{2}$ we have

$$
d\left(x_{10}, \hat{x}_{k, i}\right)= \begin{cases}2 i & \text { if } i \text { is even } \\ 2 i+1 & \text { if } i \text { is odd }\end{cases}
$$

if $k$ is odd and

$$
d\left(x_{10}, \hat{x}_{k, i}\right)= \begin{cases}2 i+1 & \text { if } i \text { is even } \\ 2 i & \text { if } i \text { is odd }\end{cases}
$$

if $k$ is even.

All of this distances give us

$$
\sum_{x \in \text { level } k} d\left(x_{10}, x\right)=2 p^{2}+k^{2}-2 k-2 p+1+H(p, k) .
$$

For other vertices we can convert those to $x_{10}$ by changing transfer vertices and apply a similar argument by choosing suitable $G_{1}$ and $G_{2}$ and compute $w_{k}$.

By a straightforward computation (if irem means the positive integer remainder) we can see:

$$
\begin{aligned}
H(p, k) & =2 p-1+\operatorname{irem}(\mathrm{k}+\mathrm{p}, 2) \\
& =2 p-1+\frac{1}{2}+\frac{1}{2}(-1)^{k-\operatorname{irem}(\mathrm{p}, 2)+1},
\end{aligned}
$$


where

$$
\operatorname{irem}(\mathrm{p}, 2)= \begin{cases}0 & \text { if } p \text { is even } \\ 1 & \text { if } p \text { is odd. }\end{cases}
$$

So, by Lemma 1 , when $1 \leq k \leq p$, we have

$$
w_{k}=2 p^{2}+k^{2}-2 k+\frac{1}{2}+\frac{1}{2}(-1)^{k-\operatorname{irem}(\mathrm{p}, 2)+1} .
$$

Also in the graph $G$,

$$
\begin{aligned}
d\left(x_{10}\right) & =\sum_{x \in \text { level } 0} d\left(x_{10}, x\right)+\sum_{x \in \text { level } 1} d\left(x_{10}, x\right)+\cdots+\sum_{x \in \text { level } q} d\left(x_{10}, x\right) \\
& =w_{1}+w_{2}+\cdots+w_{q} .
\end{aligned}
$$

So

$$
d\left(x_{10}\right)=d\left(x_{11}\right)=\cdots=d\left(x_{2 p-1,1}\right)=w_{1}+w_{2}+\cdots+w_{q} .
$$

This leads us to the following corollary:

Corollary 1. For each vertex $u$ on level 1 we have

$$
d(u)=w_{1}+w_{2}+\cdots+w_{q}
$$

Now suppose that $p>q$. Then by lemma 2 and equation (1) we have

$$
\begin{aligned}
d(u) & =\sum_{k=1}^{q}\left(2 p^{2}+k^{2}-2 k+\frac{1}{2}+\frac{1}{2}(-1)^{k-\operatorname{irem}(\mathrm{p}, 2)+1}\right) \\
& =2 p^{2} q+\frac{q^{3}}{3}-\frac{q^{2}}{2}-\frac{q}{3}+\frac{1}{4}(-1)^{-\operatorname{irem}(p, 2)+1+q}+\frac{1}{4}(-1)^{-\operatorname{irem}(p, 2)} .
\end{aligned}
$$

Also if $p \leq q$, then by Lemma 1 and equation (1) we have

$$
\begin{aligned}
d(u)= & w_{1}+w_{2}+\cdots+w_{p-1}+w_{p}+w_{p+1}+\cdots+w_{q} \\
= & \sum_{k=1}^{p-1}\left(2 p^{2}+k^{2}-2 k+\frac{1}{2}+\frac{1}{2}(-1)^{k-\operatorname{irem}(\mathrm{p}, 2)+1}\right)+ \\
& \quad \sum_{k=p}^{q} p(p+2 k-2) \\
= & \frac{p^{3}}{3}+\frac{p^{2}}{2}-\frac{p}{3}-\frac{1}{4}(-1)^{-\operatorname{irem}(p, 2)+1+p}-\frac{1}{2}-\frac{1}{4}(-1)^{-\operatorname{irem}(p, 2)+1}+p^{2} q-p q+p q^{2}
\end{aligned}
$$

We summarize the above results in the following proposition

Corollary 2. For each vertex $u$ on level $1, d(u)$ is given by

Case 1: $p$ is even

$$
d(u)= \begin{cases}2 p^{2} q+\frac{q^{3}}{3}-\frac{q^{2}}{2}-\frac{q}{3}+\frac{1}{4}+\frac{1}{4}(-1)^{q+1} & \text { if } \quad p>q \\ \frac{p}{6}\left[2 p^{2}+3 p-2+6 p q-6 q+6 q^{2}\right] & \text { if } \quad p \leq q\end{cases}
$$


Case 2: $\mathrm{p}$ is odd

$$
d(u)= \begin{cases}2 p^{2} q+\frac{q^{3}}{3}-\frac{q^{2}}{2}-\frac{q}{3}-\frac{1}{4}+\frac{1}{4}(-1)^{q} & \text { if } \quad p>q \\ \frac{p^{3}}{3}+\frac{p^{2}}{2}-\frac{p}{3}-\frac{1}{2}+p^{2} q-p q+p q^{2} & \text { if } \quad p \leq q\end{cases}
$$

Theorem 1. The Wiener index of $G:=T U V C_{6}[2 p, q]$ nanotubes is given by

Case 1: $p$ is even

$$
W(G)= \begin{cases}\frac{p}{12}\left[3(-1)^{q+1}+3+24 q^{2} p^{2}-8 q^{2}+2 q^{4}\right] & \text { if } \quad p>q \\ \frac{-p^{2}}{6}\left[8 q-4 p+p^{3}-4 q p^{2}-4 q^{3}-6 q^{2} p\right] & \text { if } \quad p \leq q\end{cases}
$$

Case 2: $\mathrm{p}$ is odd

$$
W(G)= \begin{cases}\frac{p}{12}\left[3(-1)^{q}-3+24 q^{2} p^{2}-8 q^{2}+2 q^{4}\right] & \text { if } p>q \\ \frac{-p}{6}\left[-4 p^{3} q-4 p q^{3}-6 q^{2} p^{2}+3+8 q p-4 p^{2}+p^{4}\right] & \text { if } \quad p \leq q\end{cases}
$$

Proof: See [19].

Now we are in the position to prove the main result of this section.

Theorem 2. The Schultz index of $G:=T U V C_{6}[2 p, q]$ nanotubes is given by

Case 1: $p$ is even

$$
S(G)= \begin{cases}\frac{p}{6}\left[-48 p^{2} q+72 p^{2} q^{2}+3(-1)^{q+1}+3-8 q^{3}-12 q^{2}+6 q^{4}+8 q\right] & \text { if } p>q \\ \frac{-p^{2}}{3}\left[-18 q^{2} p+3 p^{3}-6 p-12 p^{2} q-12 q^{3}+12 q+4 p^{2}-4+12 p q+12 q^{2}\right] & \text { if } p \leq q\end{cases}
$$

Case 2: $\mathrm{p}$ is odd

$$
S(G)= \begin{cases}\frac{p}{6}\left[72 q^{2} p^{2}+6 q^{4}-12 q^{2}-3+3(-1)^{q}-48 p^{2} q-8 q^{3}+8 q\right] & \text { if } p>q \\ \frac{-p}{3}\left[-12 p^{3} q-12 p q^{3}-18 p^{2} q^{2}+3+12 p q-6 p^{2}+3 p^{4}+\right. & \\ \left.4 p^{3}-4 p+12 p^{2} q+12 p q^{2}\right] & \text { if } \quad p \leq q\end{cases}
$$

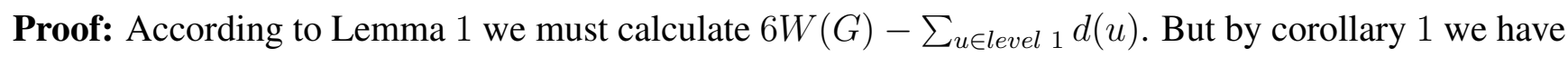

$$
d(u)=w_{1}+w_{2}+\cdots+w_{q}
$$

Since there are $2 p$ vertices on level 1 therefore

$$
S(G)=6 W(G)-4 p d(u)
$$

Finally by replacing $d(u)$ from corollary 1 in the equation (2) the result obtains. 
Table 1. Schultz index of short tubes, $p>q$.

\begin{tabular}{|l|l|l||l|l|l|}
\hline$p$ & $q$ & $S(G)$ & $p$ & $q$ & $S(G)$ \\
\hline 6 & 2 & 6912 & 5 & 2 & 4000 \\
6 & 3 & 18366 & 5 & 3 & 10650 \\
6 & 4 & 35424 & 5 & 4 & 20720 \\
6 & 5 & 58656 & 9 & 5 & 193266 \\
10 & 2 & 32000 & 9 & 6 & 288432 \\
10 & 5 & 264160 & 9 & 7 & 404514 \\
10 & 6 & 393440 & 9 & 8 & 542880 \\
10 & 7 & 550560 & 15 & 8 & 2425440 \\
10 & 8 & 736960 & 15 & 7 & 1823310 \\
10 & 9 & 954400 & 15 & 6 & 1310160 \\
\hline
\end{tabular}

Table 2. Schultz index of long tubes, $p \leq q$.

\begin{tabular}{|l|l|l||l|l|l|}
\hline$p$ & $q$ & $S(G)$ & $p$ & $q$ & $S(G)$ \\
\hline 4 & 4 & 10816 & 3 & 4 & 4752 \\
4 & 5 & 18304 & 3 & 5 & 8262 \\
4 & 6 & 28352 & 3 & 6 & 13104 \\
4 & 7 & 41344 & 3 & 7 & 19494 \\
4 & 8 & 57664 & 3 & 8 & 27648 \\
10 & 21 & 6810400 & 11 & 11 & 1954502 \\
10 & 22 & 7641600 & 11 & 12 & 2371952 \\
10 & 23 & 8536800 & 11 & 13 & 2839524 \\
10 & 24 & 9498400 & 11 & 14 & 3359312 \\
10 & 25 & 10528800 & 11 & 15 & 3935030 \\
\hline
\end{tabular}




\section{Experimental Section}

Tables 1 and 2 show the numerical data for the Schultz index in tubes $T U V C_{6}[2 p, q]$ of various dimensions.

\section{Acknowledgements}

This work was supported by a grant from the Center of Research of Islamic Azad University, Najafabad Branch, Isfahan, Iran.

\section{References and Notes}

1. Wiener, H. Structural Determination of Paraffin Boiling points. J. Am. Chem. Soc. 1947, 69, 17-20.

2. Wiener, H. Correlation of Heats of Isomerization and differences in heats of vaporization of isomers among the paraffin Hydrocarbons. J. Am. Chem. Soc. 1947, 69, 2636-2638.

3. Wiener, H. Influence of Interatomic Forces on Paraffin Properties. J. Chem. Phys. 1947, 15, 766766.

4. Balaban, A. T.; Devillers, J. Eds. Topological Indices and Related Descriptors in QSAR and QSPR; Gordon and Breach: Amsterdam, 1999.

5. Diudea, M. V. Indices of Reciprocal Properties or Harary Indices. J. Chem. Inf. Comput. Sci. 1997, 37, 292-299.

6. Gutman, I.; Polansky, O.E. Mathematical Concepts in Organic Chemistry; Springer: Berlin, 1986.

7. Gutman, I. Relation Between Hyper-Wiener and Wiener Index. Chem. Phys. Lett. 2002, 364, 352-356.

8. Gutman, I.; Furtula, B. Hyper-Wiener Index vs. Wiener index. Monatshefte für Chemie 2003, 134, 975-981.

9. Randić, M.; Trinajstić, N. In Search for Graph Invariants of Chemical Interest. J. Mol. Struct. (THEOCHEM) 1993, 300, 551-571.

10. Todeschini, R.; Consonni, V. Handbook of Molecular Descriptors; Wiley: Weinheim, 2000.

11. Schultz, H. P. Topological organic chemistry 1. Graph Theory and Topological Indices of Alkanes. J. Chem. Inf. Comput. Sci. 1989, 29, 227-228.

12. Mihalić, Z.; Nikolić, S.; Trinajstić, N. Comparative Study of Molecular Descriptors Derived from the Distance Matrix. J. Chem. Inf. Comput. Sci. 1992, 32, 28-37.

13. Müller, W. R.; Szymanski, K.; Knop, J. V.; Trinajstić, N. Mulecular Topological Index. J. Chem. Inf. Comput. Sci. 1990, 30, 160-163.

14. Schultz, H. P.; Schultz, T. P. Topological Organic Chemistry. 6. Graph Theory and Topological Indices of Cycloalkanes. J. Chem. Inf. Comput. Sci. 1993, 33, 240-243.

15. Schultz, H. P.; Schultz, T. P. Topological Organic Chemistry. 7. Graph Theory and Topological Indices of Cycloalkanes. J. Chem. Inf. Comput. Sci. 1993, 33, 863-867.

16. Gutman, I.; Klavžar, S. Comparison of the Schultz Molecular Topological Index with the Wiener Index. J. Chem. Inf. Comput. Sci. 1996, 36, 1001-1003.

17. Gutman, I.; Klavžar, S. Bounds for the Schultz Molecular Topological Index of Benzenoid Systems in Terms of the Wiener Index. J. Chem. Inf. Comput. Sci. 1997, 37, 741-744. 
18. Plavšić, D.; Nikolić, S.; Trinajstić, N.; Klein, D.J. Relation between the Wiener Index and the Schultz Index for Several Classes of Chemical Graphs. Croat. Chem. Acta 1993, 66, 345-353.

19. Diudea, M. V.; Stefu, M.; Parv, B.; John, P. E. Wiener Index of Armchair Polyhex Nanotubes. Croat. Chem. Acta 2004, 77, 111-115.

20. Ping Lu, J. Elastic Properties of Carbon Nanotubes and Nanoropes. Phys. Rev. Lett. 1997, 79, 1297-1300.

21. Wong, E. W.; Sheehan, P. E.; Lieber, C.M. Nanobeam mechanics: Elasticity, strength, and toughness of nanorods and nanotubes. Science 1997, 277, 1971-1975.

22. Heck, A. Introduction to Maple, $3^{\text {rd }}$ Ed. Springer-Verlag: New York, 2003.

\section{Appendix}

The following code is the MAPLE program [22] used to produce the graph of $T U H C_{6}[2 p, q]$ and to compute the Schultz index of the graph.

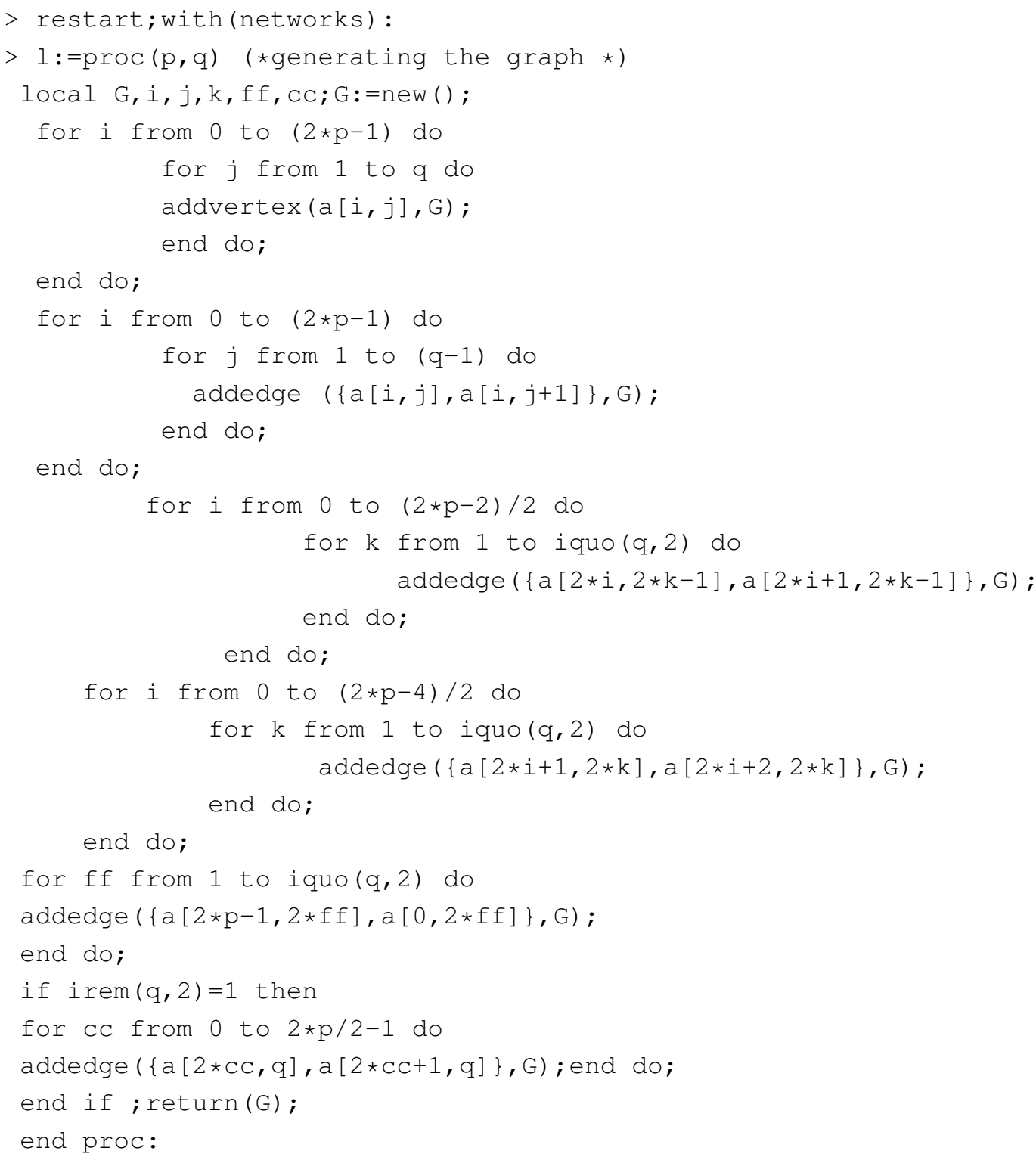




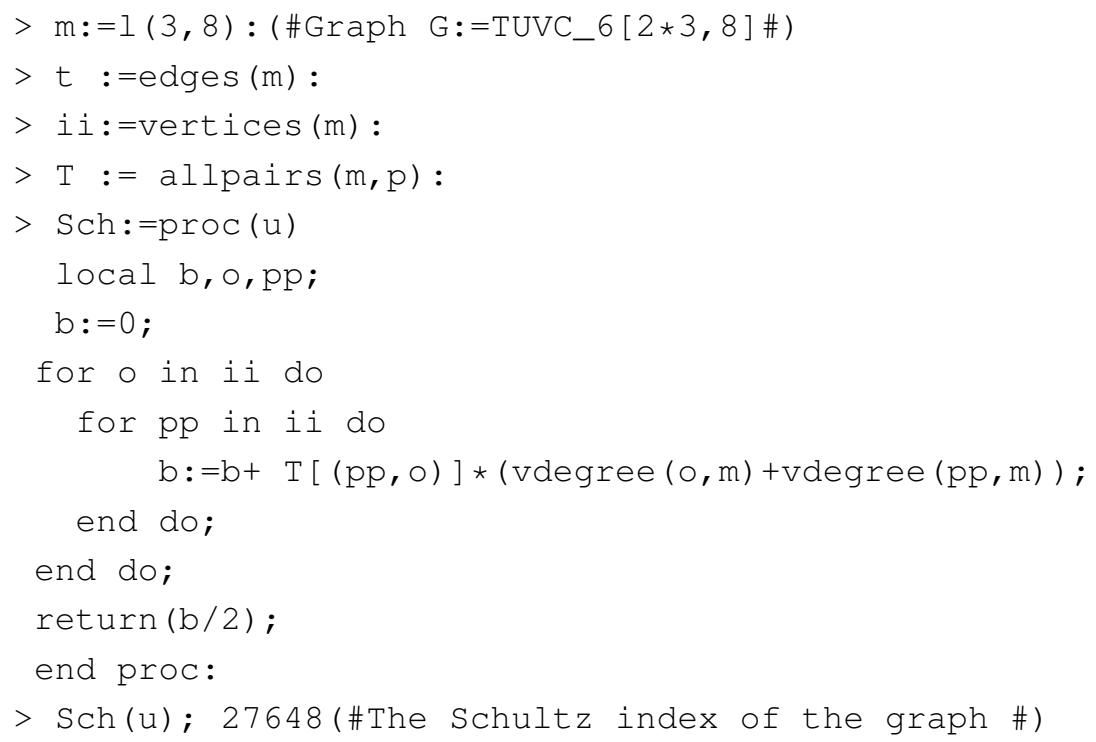

(c) 2008 by the authors; licensee Molecular Diversity Preservation International, Basel, Switzerland. This article is an open-access article distributed under the terms and conditions of the Creative Commons Attribution license (http://creativecommons.org/licenses/by/3.0/). 